\title{
Efficacy of tyrosine kinase inhibitors in non-small-cell lung cancer patients undergoing dose reduction and those with a low body surface area
}

\author{
SHINYA SATO ${ }^{1}$, KOICHI KURISHIMA ${ }^{2}$, KUNIHIKO MIYAZAKI ${ }^{1}$, TAKAHIDE KODAMA ${ }^{1}$, \\ HIROICHI ISHIKAWA ${ }^{3}$, KATSUNORI KAGOHASHI ${ }^{4}$, TOMOHIRO TAMURA ${ }^{2}$, \\ SHINSUKE HOMMA ${ }^{2}$, HIROAKI SATOH ${ }^{4}$ and NOBUYUKI HIZAWA ${ }^{2}$
}

\author{
${ }^{1}$ Division of Respiratory Medicine, Ryugasaki Saiseikai General Hospital, Ryugasaki, Ibaraki 301-0854; \\ ${ }^{2}$ Division of Respiratory Medicine, Faculty of Clinical Medicine, University of Tsukuba, Tsukuba, Ibaraki 305-8575; \\ ${ }^{3}$ Division of Respiratory Medicine, Tsukuba Medical Center Hospital and Regional Cancer Center, Tsukuba, Ibaraki 305-8558; \\ ${ }^{4}$ Division of Respiratory Medicine, Mito Medical Center, University of Tsukuba, Mito, Ibaraki 310-0015, Japan
}

Received January 27, 2014; Accepted April 8, 2014

DOI: $10.3892 / \mathrm{mco} .2014 .281$

\begin{abstract}
The objective of this study was to evaluate the efficacy of epidermal growth factor receptor-tyrosine kinase inhibitors (EGFR-TKIs) in patients undergoing dose reduction and in those with a low body surface area (BSA). The association between dose reduction, low BSA and efficacy, including response rate (RR), disease control rate (DCR), progression-free survival (PFS) and overall survival (OS), were evaluated in patients prescribed TKIs between September, 2002 and May, 2013. A total of 282 patients received EGFR-TKIs during the study period, $53(18.8 \%)$ of whom underwent a dose reduction (21.4 and $31.6 \%$ of the patients with a BSA of $<1.5$ and $<1.25 \mathrm{~m}^{2}$, respectively). Eleven $(20.8 \%)$ of these 53 patients had a dose reduction due to adverse events (AEs) $>$ grade 3 . In either gefitinib or erlotinib treatment, the RR, DCR, PFS and OS in EGFR-mutated patients with a BSA of $<1.5 \mathrm{~m}^{2}$ were not different from those in patients with a BSA of $>1.5 \mathrm{~m}^{2}$. In addition, there were no differences in these parameters between patients with and those without a dose reduction of TKIs. The dose of TKIs in patients with AEs and in those with low BSA should be determined with caution. To confirm the equal efficacy of TKIs in patients undergoing a dose reduction, prospective observational studies with less patient heterogeneity are required.
\end{abstract}

Correspondence to: Professor Hiroaki Satoh, Division of Respiratory Medicine, Mito Medical Center, University of Tsukuba, 3-2-7 Miya-machi, Mito, Ibaraki 310-0015, Japan

E-mail: hirosato@md.tsukuba.ac.jp

Key words: body surface area, dose reduction, epidermal growth factor receptor-tyrosine kinase inhibitor, gefitinib, erlotinib, non-small-cell lung cancer

\section{Introduction}

The recommended dose of traditional cytotoxic antitumor agents is generally determined at or near their maximum-tolerated dose (1-3). However, in the case of epidermal growth factor receptor-tyrosine kinase inhibitors (EGFR-TKIs), the recommended dose is determined at an optimal biological dose to minimize the risk of adverse events (AEs) without compromising efficacy (4-6). However, body size was not taken into consideration in previous dose-finding studies. A significant number of EGFR-mutated patients are female and, in their majority, the body surface area (BSA) may be low (7-9). Therefore, a dose reduction due to AEs or low BSA may be required in daily clinical practice for such patients. However, there is limited information on the association between low BSA and TKI dose reduction and efficacy (10). In addition, the efficacy of TKIs in patients undergoing a dose reduction due to severe AEs has not been clearly determined. In the present study, we evaluated the efficacy of TKIs in patients undergoing a dose reduction due to AEs and in those with a low BSA in clinical practice.

\section{Patients and methods}

Patients. A total of 282 consecutive patients with pathologically confirmed non-small-cell lung cancer (NSCLC), who were treated with TKIs at the University of Tsukuba Hospital, the Tsukuba Medical Center Hospital, the Ryugasaki Saiseikai General Hospital and the Mito Medical Center-Mito Kyodo General Hospital between September, 2005 and May, 2013, were retrospectively analyzed. The histopathological diagnoses were performed according to the World Health Organization classification system (11) and the patients were staged according to the Union for International Cancer Control tumor node metastasis staging system (12).

The patient characteristics, efficacy and safety were evaluated using patient data extracted from the database of each participating institution. 
Tumor response. Tumor responses were classified as complete response, partial response, stable disease, progressive disease or not evaluable, according to the Response Evaluation Criteria in Solid Tumors, version 1.1 (13). In this study, we defined 'dose reduction' of TKIs was as a reduction of the starting dose, as well as a reduction during the course of treatment. Patients undergoing discontinuation of TKIs without any dose reduction were not included. The effect of BSA on progression-free survival (PFS) and overall survival (OS) was evaluated. The effect of TKIs, with or without dose reduction, was also evaluated in the analysis of PFS and OS. The present retrospective study conformed to the Ethical Guidelines for Clinical Studies issued by the Ministry of Health, Labor and Welfare of Japan.

Statistical analysis. Statistical significance between two groups was determined using the Mann-Whitney U test and the Chi-square test. The patient survival time was calculated from the day of TKI initiation to death or last follow-up. The survival rate was analyzed with the Kaplan-Meier method and comparisons were performed using the log-rank test. $\mathrm{P}<0.05$ was considered to indicate a statistically significant difference.

\section{Results}

Patient characteristics. During the study period, a total of 282 patients were treated with TKIs (213 with gefitinib and 69 with erlotinib). The median age was 66 years (range, 21-90 years). The patient characteristics are summarized in Table I. A total of 124 patients $(44.0 \%)$ were male, $128(45.4 \%)$ had smoking history and 197 (69.9\%) had good performance status (0-1). The tumors were classified as 243 (86.2\%) adenocarcinomas, 35 (12.4\%) squamous cell carcinomas and $4(1.4 \%)$ other types. Nineteen patients $(6.7 \%)$ had a low BSA $\left(<1.25 \mathrm{~m}^{2}\right)$ and $112(39.7 \%)$ had $1.25<\mathrm{BSA}<1.5 \mathrm{~m}^{2}$. A dose reduction of TKIs for any reason was performed in 53 (18.8\%) of the 282 patients (12.2\% receiving gefitinib and $39.1 \%$ erlotinib). Of these, 21.4 and $31.6 \%$ with a BSA of $<1.5$ and $<1.25 \mathrm{~m}^{2}$, respectively, underwent a dose reduction of TKIs.

Overall response rate $(R R)$, disease control rate $(D C R)$, PFS and AEs. In the 282 patients, including 96 (34.1\%) with mutated-type EGFR, 52 (18.4\%) with wild-type EGFR and $134(47.5 \%)$ with unknown EGFR status, the objective RR was $40.1 \%$, the DCR was $62.1 \%$ and the PFS was 4.2 months. Of the 282 patients, $23(8.2 \%)$ developed AEs $>$ grade 3 . The most common AEs were severe skin reaction and liver dysfunction.

AEs, BSA and dose reduction in patients treated with gefitinib. Of the 213 patients receiving gefitinib, 16 exhibited AEs $>$ grade 3 . Among these, 6 (37.5\%) underwent a dose reduction. However, of the 197 patients who did not have AEs > grade 3, $20(10.2 \%)$ received a dose reduction. The difference was statistically significant $(\mathrm{P}=0.006)$. The proportion of the patients undergoing a dose reduction was not associated with BSA in those with or without AEs $>$ grade 3 (Table II).

AEs, BSA and dose reduction in patients treated with erlotinib. Of the 69 patients receiving erlotinib therapy, 7 developed AEs > grade 3. Among these, $5(71.4 \%)$ had a dose reduction. Of the 62 patients who did not have AEs > grade 3, $22(35.5 \%)$
Table I. Characteristics of 282 patients treated with TKIs.

\begin{tabular}{lc}
\hline Characteristics & No. $(\%)$ \\
\hline Age, years (median, range) & $66,21-90$ \\
Gender (male/female) & $124(44.0) / 158(56.0)$ \\
Smoking habit (present/absent) & $128(45.4) / 154(54.6)$ \\
Performance status (0-1/2-4) & $197(69.9) / 85(30.1)$ \\
Pathology & \\
Adenocarcinoma & $243(86.2)$ \\
Squamous cell carcinoma & $35(12.4)$ \\
Other & $4(1.4)$ \\
EGFR mutation type & \\
Mutated & $96(34.1)$ \\
Wild-type & $52(18.4)$ \\
Unknown & $134(47.5)$ \\
Body surface area $\left(\mathrm{m}^{2}\right)$ & \\
$<1.25$ & $19(6.7)$ \\
$1.25-1.50$ & $112(39.7)$ \\
$>1.50$ & $151(53.6)$ \\
\hline
\end{tabular}

TKIs, tyrosine kinase inhibitors; EGFR, epidermal growth factor receptor.

received a dose reduction. The difference was not statistically significant $(\mathrm{P}=0.162)$. The proportion of patients undergoing a dose reduction was not associated with BSA in those with or those without AEs > grade 3 (Table III).

BSA and RR, DCR, PFS and OS in EGFR-mutated patients treated with gefitinib. We compared RR, DCR and PFS between 45 patients with $\mathrm{BSA}>1.5 \mathrm{~m}^{2}$ and 37 with $\mathrm{BSA}<1.5 \mathrm{~m}^{2}$. The RR and DCR were 82.2 and $95.6 \%$ in the former patient group and 67.6 and $91.9 \%$ in the latter group, respectively. There was no statistically significant difference between the two groups $(\mathrm{P}=0.196$ and 0.645 , respectively). There was also no statistically significant difference in the PFS between the two groups of patients (BSA $>1.5 \mathrm{~m}^{2}, 12.1$ months vs. $<1.5 \mathrm{~m}^{2}, 12.8$ months; $\mathrm{P}=0.861$ ) (Fig. 1). The OS appeared to be longer in patients with $\mathrm{BSA}<1.5 \mathrm{~m}^{2}$ compared to that in patients with $>1.5 \mathrm{~m}^{2}$, although the difference was not statistically significant $(\mathrm{P}=0.080)$.

Dose reduction and RR, DCR, PFS and $O S$ in EGFR-mutated patients treated with gefitinib. We next evaluated the effect of dose reduction of gefitinib on RR, DCR, PFS and OS. There were 19 patients with and 63 without a dose reduction of gefitinib. The RR and DCR were 78.9 and $94.7 \%$ in the former group of patients and 74.6 and $93.7 \%$ in the latter group, respectively. There was no statistically significant difference between the two groups $(\mathrm{P}=0.699$ and 0.862 , respectively). As shown in Fig. 2, there was also no statistically significant difference in PFS between patients with and those without dose reduction (12.1 vs. 12.3 months, respectively; $\mathrm{P}=0.522$ ). There appeared to be a difference in OS between patients with and those without dose reduction, but the difference was not statistically significant $(\mathrm{P}=0.154)$. 
Table II. Adverse events (AEs) > grade 3, body surface area (BSA) and dose reduction in 213 patients treated with gefitinib.

\begin{tabular}{|c|c|c|c|c|}
\hline Patients & No. & $\operatorname{BSA}\left(\mathrm{m}^{2}\right)$ & $\begin{array}{l}\text { Patients } \\
\text { with dose } \\
\text { reduction } \\
\text { no. }(\%)\end{array}$ & $\begin{array}{l}\text { Patients } \\
\text { without dose } \\
\text { reduction } \\
\text { no. }(\%)\end{array}$ \\
\hline \multirow[t]{4}{*}{ With AEs } & 16 & & $6(37.5 \%)$ & $10(62.5 \%)$ \\
\hline & & $<1.25$ & 0 & 0 \\
\hline & & $1.25-1.5$ & 2 & 7 \\
\hline & & $>1.5$ & 4 & 3 \\
\hline \multirow[t]{4}{*}{ Without AEs } & 197 & & $20(10.2 \%)$ & $177(89.2 \%)$ \\
\hline & & $<1.25$ & 3 & 11 \\
\hline & & $1.25-1.5$ & 12 & 69 \\
\hline & & $>1.5$ & 5 & 97 \\
\hline
\end{tabular}

Table III. Adverse events (AEs) > grade 3, body surface area (BSA) and dose reduction in 69 patients treated with erlotinib.

\begin{tabular}{|c|c|c|c|c|}
\hline Patients & No. & $\operatorname{BSA}\left(m^{2}\right)$ & $\begin{array}{l}\text { Patients } \\
\text { with dose } \\
\text { reduction } \\
\text { no. }(\%)\end{array}$ & $\begin{array}{l}\text { Patients } \\
\text { without dose } \\
\text { reduction } \\
\text { no. }(\%)\end{array}$ \\
\hline \multirow[t]{4}{*}{ With AEs } & 7 & & $5(71.4 \%)$ & $2(28.6 \%)$ \\
\hline & & $<1.25$ & 0 & 0 \\
\hline & & $1.25-1.5$ & 1 & 0 \\
\hline & & $>1.5$ & 4 & 2 \\
\hline \multirow[t]{4}{*}{ Without AEs } & 62 & & $22(35.5 \%)$ & $40(64.5 \%)$ \\
\hline & & $<1.25$ & 3 & 2 \\
\hline & & $1.25-1.5$ & 7 & 14 \\
\hline & & $>1.5$ & 12 & 24 \\
\hline
\end{tabular}

The association among BSA, dose reduction of gefitinib, PFS and OS is shown in Fig. 3. Of the 24 patients with BSA $<1.5 \mathrm{~m}^{2}, 22(91.7 \%)$ were female, $13(54.2 \%)$ were aged $\geq 75$ years, $10(41.7 \%)$ underwent a dose reduction and $19(79.2 \%)$ received no further cytotoxic antitumor chemotherapy. There were no statistical differences among these variables.

$B S A$ and $R R, D C R, P F S$ and $O S$ in patients treated with erlotinib. In the 14 EGFR-mutated patients treated with erlotinib, there was no statistically significant difference in RR, DCR and PFS between those with and those without dose reduction (RR: 87.5 vs. $50 \%, \mathrm{P}=0.124$; DCR: 100 vs. $89.3 \%, \mathrm{P}=0.230$; and PFS: 14.3 vs. 8.0 months, $\mathrm{P}=0.409$ ).

In the 39 wild-type EGFR patients treated with erlotinib, there was no statistically significant difference in PFS between those with and those without dose reduction $(\mathrm{P}=0.794)$. There was no apparent statistical difference in OS between patients with a BSA of $>1.5$ and those with a BSA of $<1.5 \mathrm{~m}^{2}(\mathrm{P}=0.589)$. However, there was a statistically significant difference in OS between those with and those without dose reduction $(\mathrm{P}=0.172)$.

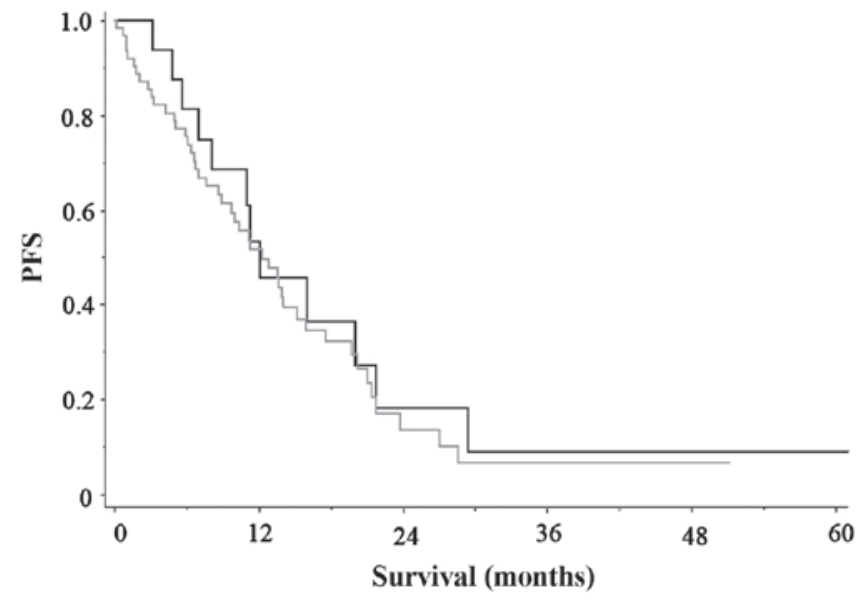

Figure 1. Progression-free survival (PFS) curves in gefitinib-treated epidermal growth factor receptor-mutated patients with a body surface area (BSA) of $>1.5 \mathrm{~m}^{2}$ (gray line) and those with a BSA of $<1.5 \mathrm{~m}^{2}$ (black line).

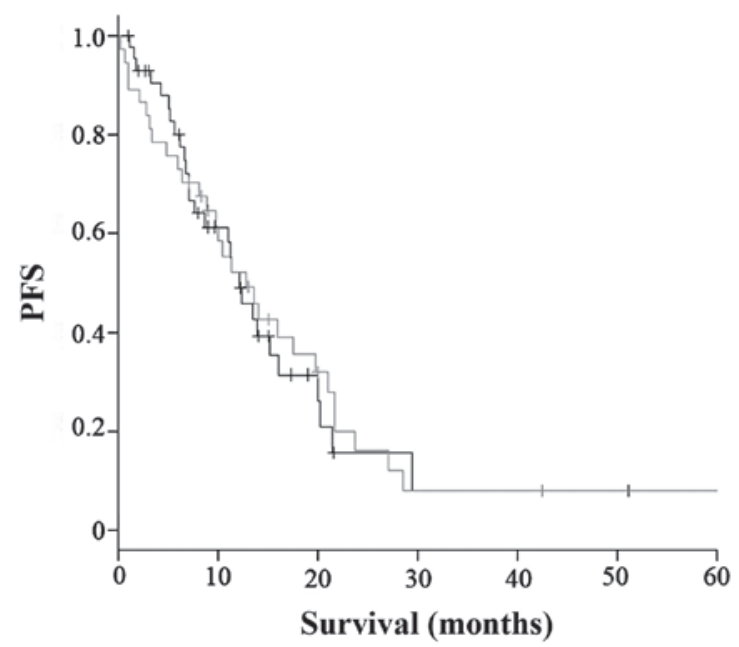

Figure 2. Progression-free survival (PFS) curves in gefitinib-treated epidermal growth factor receptor-mutated patients undergoing dose reduction (gray line) and those without dose reduction (black line).

\section{Discussion}

Previous clinical trials reported that using a fixed dose of EGFR-TKIs achieved a significant improvement in PFS with acceptable AE profiles $(14,15)$. Either gefitinib or erlotinib are currently among the first-choice treatments for advanced NSCLC patients with EGFR mutation $(14,15)$. However, the efficacy of TKIs in the clinical setting, particularly in patients undergoing a dose reduction due to toxicity or low BSA, remains unclear. There remained the question whether a strictly fixed dose of TKIs, determined by dose-finding studies, would exhibit the same efficacy in patients undergoing a dose reduction as in those without a dose reduction. Therefore, we evaluated RR, DCR, PFS and OS in patients with and those without dose reduction of TKIs. In the present study, clinically relevant AEs >grade 3 were observed in $23(8.2 \%)$ of the 282 patients, leading to a dose reduction in 


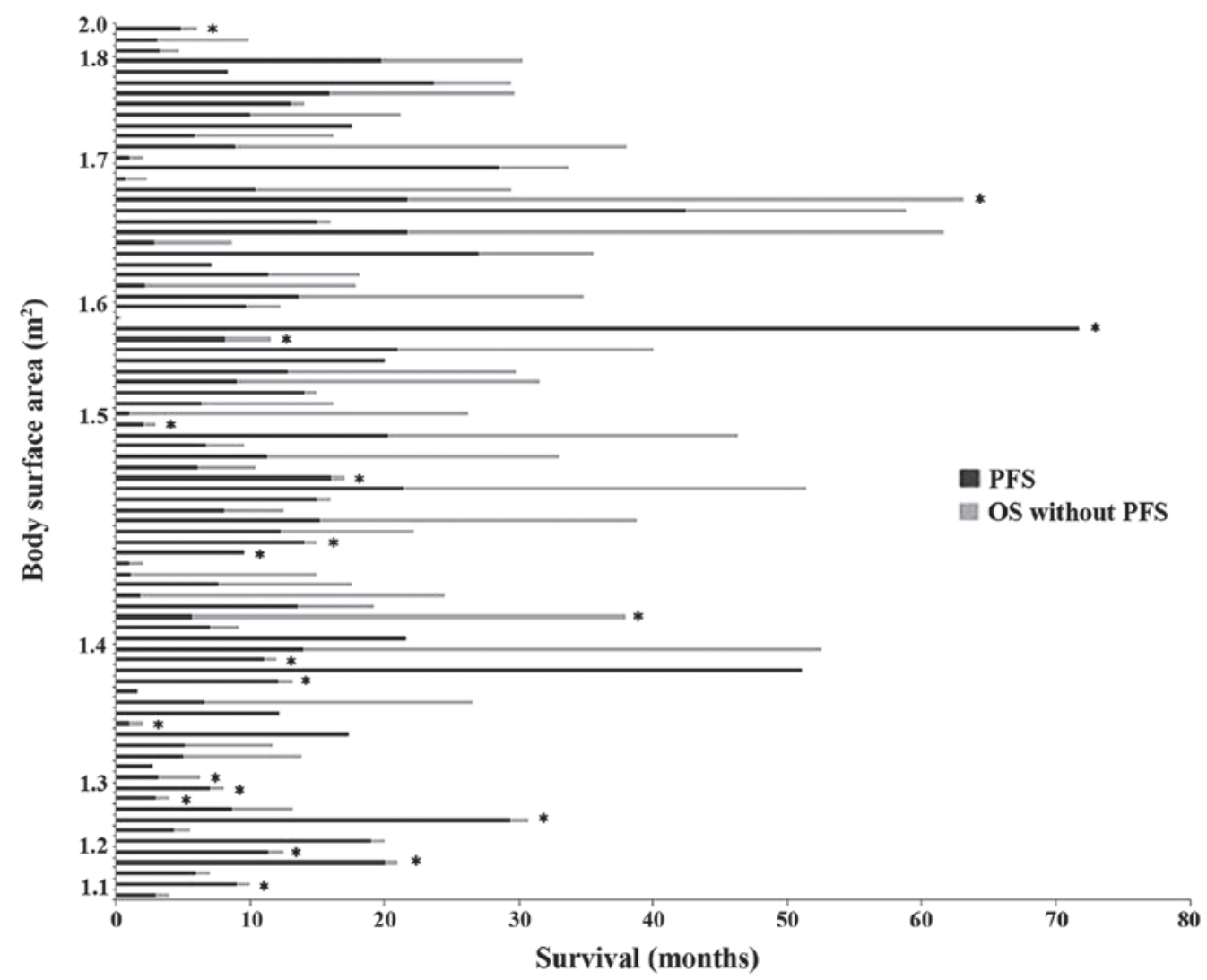

Figure 3. Body surface area and dose reduction of gefitinib in EGFR-mutated patients. "Patient with dose reduction of gefitinib. PFS, progression-free survival; OS, overall survival; EGFR, epidermal growth factor receptor.

11 of the patients. Therefore, 42 patients underwent a dose reduction due to reasons other than AEs. In addition, $46.4 \%$ of the 282 patients had a BSA of $<1.5 \mathrm{~m}^{2}$, demonstrating that a considerable percentage of patients with low BSA received TKI therapy, which may be associated with the fact that a number of those patients were EGFR-mutated female patients. In fact, in previous reports on treatment with TKIs, either in clinical trials or clinical practice, there was a higher population of female patients with NSCLC compared to that in the general population $(7-9,16)$.

Our study did not identify any disadvantage regarding PFS from a dose reduction in patients receiving gefitinib or erlotinib. However, as these findings were obtained from a relatively small number of patients in a retrospective study, we were unable to draw definitive conclusions on how dose reduction would affect patients with AEs and those with low BSA. We recommend that our results be meaningfully interpreted rather than be overlooked as anecdotal findings. We may need to reconsider the most appropriate dosage of TKIs for such patients, in order to obtain maximum efficacy with an acceptable toxicity profile.

To the best of our knowledge, there has been only one previous study evaluating the effect of BSA on the efficacy of TKIs (10). That study investigated the potential effect of BSA on the PFS and OS of patients with advanced EGFR-mutated NSCLC who were treated with gefitinib (10) and found that BSA affected the outcome of gefitinib therapy, with a higher BSA being associated with worse PFS (10). The main focus of that study was treatment efficacy in patients with a high
BSA; by contrast, our interest was not only the effects of dose reduction in patients with AEs as well as in patients with a low BSA. In the previous study, the PFS of patients with low BSA with treatment interruption or reduced dose was either equal to or superior to that of all low-BSA patients (10), which was consistent with our results. Ichihara et al (10) hypothesized that the patients who required a dose reduction due to AEs may be those with relatively high blood concentrations of the agent, due to factors such as drug metabolism. This hypothesis requires confirmation in future studies.

Another point investigated was the effect of BSA on OS. Ichihara et al (10) reported that OS was not associated with BSA in their study. In our study, there was no apparent difference in OS between patients with BSA $<1.5 \mathrm{~m}^{2}$ and those with $\mathrm{BSA}>1.5 \mathrm{~m}^{2}$ receiving treatment with either gefitinib or erlotinib. The OS in patients without dose reduction appeared to be longer compared to that in patients with dose reduction, but the difference was not statistically significant. As suggested by Ichihara et al (10), a possible reason may be the effect of preceding and/or subsequent therapies on OS. In their study, as many as $70 \%$ of the patients received chemotherapy following disease progression post-gefitinib monotherapy, suggesting that post-progression therapy may have blunted any difference in PFS between the high- and low-BSA subgroups (10). Another explanation for these results may be the fact that further cytotoxic antitumor chemotherapy was not indicated in the majority of the low-BSA patients, as observed in our study. We were unable to identify a statistically significant difference in dose reduction for patients with low BSA. 
The analysis of our data set and conclusions drawn were limited by the small sample size, retrospective design and heterogeneity in the EGFR mutations, TKIs used, clinicians' experience with TKIs, reasons for dose reduction and exclusion of patients with discontinuation of TKIs without any dose reduction. Another major limitation is that pharmacokinetic data were not included in this analysis. It remains unclear whether BSA differences led to inter-patient pharmacokinetic variability, resulting in the observed difference in PFS. A pharmacokinetics-pharmacodynamics study is required to clarify this issue. The blood concentration of cytotoxic agents is closely associated with their efficacy (17-19). Similarly, the blood concentration of TKIs also appears to be associated with their efficacy $(20,21)$.

Dose-reduction estimation studies for TKIs may be crucial, particularly for patients with low BSA. Heterogeneity in these factors should be taken into consideration, or exclude causes of heterogeneity. Prospective studies investigating the incidence of dose reduction in patients with AEs and those with low BSA may be meaningful for common clinical practice. This approach may further elucidate the clinical meaning of dose reduction of TKIs in such patients.

\section{References}

1. Seymour L and Eisenhauer E: A review of dose-limiting events in phase I trials: antimetabolites show unpredictable relationships between dose and toxicity. Cancer Chemother Pharmacol 47: 2-10, 2001.

2. Hoekstra R, Verweij J and Eskens FA: Clinical trial design for target specific anticancer agents. Invest New Drugs 21: 243-250, 2003.

3. Zohar S, Lian Q, Levy V, Cheung K, Ivanova A and Chevret S: Quality assessment of phase I dose-finding cancer trials: proposal of a checklist. Clin Trials 5: 478-485, 2008.

4. Wolf M, Swaisland H and Averbuch S: Development of the novel biologically targeted anticancer agent gefitinib: determining the optimum dose for clinical efficacy. Clin Cancer Res 10: 4607-4613, 2004.

5. Takimoto CH: Maximum tolerated dose: clinical endpoint for a bygone era? Target Oncol 4: 143-147, 2009.

6. Yamamoto N, Horiike A, Fujisaka Y, et al: Phase I dose-finding and pharmacokinetic study of the oral epidermal growth factor receptor tyrosine kinase inhibitor Ro50-8231 (erlotinib) in Japanese patients with solid tumors. Cancer Chemother Pharmacol 61: 489-496, 2008.
7. Boyer M, Horwood K, Pavlakis N, et al: Efficacy of erlotinib in patients with advanced non-small-cell lung cancer (NSCLC): analysis of the Australian subpopulation of the TRUST study. Asia Pac J Clin Oncol 8: 248-254, 2012.

8. Gahr S, Stoehr R, Geissinger E, et al: EGFR mutational status in a large series of Caucasian European NSCLC patients: data from daily practice. Br J Cancer 109: 1821-1828, 2013.

9. Kaburagi T, Satoh H, Hayashihara K, et al: Observational study on the efficacy and safety of erlotinib in patients with non-small cell lung cancer. Oncol Lett 5: 435-439, 2013.

10. Ichihara $\mathrm{E}$, Hotta $\mathrm{K}$, Takigawa $\mathrm{N}$, et al: Impact of physical size on gefitinib efficacy in patients with non-small cell lung cancer harboring EGFR mutations. Lung Cancer 81: 435-439, 2013.

11. Travis WD, Brambilla E, Muller-Hermelink HK and Harris CC (eds): Pathology and Genetics: Tumours of the Lung, Pleura, Thymus and Heart. IARC Press, Lyon, pp 9-122, 2004.

12. Kligerman $\mathrm{S}$ and Abbott $\mathrm{G}$ : A radiologic review of the new TNM classification for lung cancer. AJR Am J Roentgenol 194: 562-573, 2010.

13. Sohaib A: RECIST rules. Cancer Imaging 12: 345-346, 2012.

14. Pennell NA: Integration of EGFR inhibitors and conventional chemotherapy in the treatment of non-small-cell lung cancer. Clin Lung Cancer 12: 350-359, 2011.

15. Roengvoraphoj M, Tsongalis GJ, Dragnev KH and Rigas JR: Epidermal grow th factor receptor tyrosine kinase inhibitors as initial therapy for non-small cell lung cancer: focus on epidermal growth factor receptor mutation testing and mutation-positive patients. Cancer Treat Rev 39: 839-850, 2013.

16. Hayashibara K, Satoh H, Shinohara Y, et al: A population-based study of gefitinib in patients with non-small cell lung cancer. Med Oncol 26: 222-227, 2009.

17. Miles DW, Chan A, Dirix LY, et al: Phase III study of bevacizumab plus docetaxel compared with placebo plus docetaxel for the first-line treatment of human epidermal growth factor receptor 2-negative metastatic breast cancer. J Clin Oncol 28: 3239-3247, 2010.

18. Wittenburg LA and Gustafson DL: Optimizing preclinical study design in oncology research. Chem Biol Interact 190: 73-78, 2011.

19. Fujita KI and Sasaki Y: Optimization of cancer chemotherapy on the basis of pharmacokinetics and pharmacodynamics: from patients enrolled in 'clinical trials' to those in the 'real world'. Drug Metab Pharmacokinet 29: 20-28, 2014.

20. Fukudo M, Ikemi Y, Togashi Y, et al: Population pharmacokinetics/pharmacodynamics of erlotinib and pharmacogenomic analysis of plasma and cerebrospinal fluid drug concentrations in Japanese patients with non-small cell lung cancer. Clin Pharmacokinet 52: 593-609, 2013.

21. Ranson M and Wardell S: Gefitinib, a novel, orally administered agent for the treatment of cancer. J Clin Pharm Ther 29: 95-103, 2004. 